\title{
Pattern Matching for Building Feature Extraction
}

\author{
Eva Lagunas, Member, IEEE, Moeness G. Amin, Fellow, IEEE, Fauzia Ahmad, Senior Member, IEEE, and \\ Montse Nájar, Senior Member, IEEE
}

\begin{abstract}
We address the problem of detecting building dominant scatterers using a reduced number of measurements with applications to through-the-wall radar (TWR) and urban sensing. We consider oblique illumination, which specially enhances the radar returns from the corners formed by the orthogonal intersection of two walls. This letter uses a novel type of image descriptor, named correlogram, which encodes information about spatial correlation of complex amplitudes of each TWR image pixel. The proposed technique compares the known correlogram of the scattering response of an isolated canonical corner reflector with the correlogram of the received radar signal. The feature-based nature of the proposed detector enables corner separation from other indoor scatterers, such as humans.
\end{abstract}

Index Terms-Building dominant scatterers, pattern matching, through-the-wall radar imaging (TWRI).

\section{INTRODUCTION}

$\mathbf{S}$ ENSING through building walls using radio-frequency signals to gain vision into concealed scenes is the aim of through-the-wall radar imaging (TWRI) [1]-[4]. The ability to remotely and reliably detect the presence of humans and objects of interest through opaque structures has numerous applications in civilian, law enforcement, and military sectors [5]. TWR obtains 2-D or 3-D images of the region of interest behind the front wall by combining the radar returns received at several different antenna locations along an array aperture, either real or synthesized.

In this letter, we address the problem of detecting building interior structures using a reduced number of measurements for TWRI applications. Doppler signatures or change detection techniques [6]-[8] cannot be applied since the targets of interest and clutter are both of the same stationary nature. Stationary target detection is typically performed subsequent to image formation [2]. Unlike the majority of the image-domain feature

Manuscript received November 21, 2013; revised April 5, 2014; accepted May 7, 2014. The work of E. Lagunas and M. Nájar was supported in part by the Spanish Ministry of Science and Innovation under Project TEC2011-29006C03-02 (GRE3N-LINK-MAC), by the European Commission in the framework of the Seventh Framework Programme Network of Excellence in Wireless Communications under Grant 318306, and by the Government of Catalonia under Grant 2009 SGR 891 and Grant 2014 SGR 60. The work of M. G. Amin and F. Ahmad was supported by the U.S. Army Research Office/Army Research Laboratory under Contract W911NF-11-1-0536.

E. Lagunas and M. Nájar are with the Department of Signal Theory and Communications, Universitat Politècnica de Catalunya, 08034 Barcelona, Spain (e-mail: eva.lagunas@upc.edu; montse.najar@upc.edu).

M. G. Amin and F. Ahmad are with the Center for Advanced Communications, Villanova University, Villanova, PA 19085 USA (e-mail: moeness.amin@ villanova.edu; fauzia.ahmad@villanova.edu).

Color versions of one or more of the figures in this paper are available online at http://ieeexplore.ieee.org.

Digital Object Identifier 10.1109/LGRS.2014.2324611 detection methods, the proposed approach exploits prior information of building construction practices. The building layout is usually composed of exterior and interior walls, which are parallel or perpendicular to each other. We assume flexibility in radar operation, which allows proper angular illuminations, thereby avoiding the front wall returns and preserving the corner features created by the wall junction. This can be achieved using squint beamforming or broadside beams with tilted aperture [9]. Estimating locations of dominant scatterers, such as corners, allows the inference of building interior structure. This same idea was exploited in [10] and [11], where a building-feature-based approach was applied to estimate the type and location of different canonical scattering mechanisms considering the availability of the complete volume of data.

This letter proposes an image pattern matching strategy, which is based on a novel type of image descriptor, namely, the correlogram [12]. The correlogram proposed in this letter encodes information about spatial correlation of the image complex amplitudes. The basic detection strategy adopted here is to compare the known correlogram of the scattering response of an isolated canonical corner reflector with the correlogram of the received radar returns. The correlation matching procedure can be applied using compressed observations. As such, it is a welcome alternate approach to the $L_{1}$-norm constrained optimization encountered in conventional compressive sensing (CS). The feature-based nature of the proposed detector enables corner separation from other indoor scatterers, such as humans. Numerical electromagnetic (EM) data are employed to show that the use of spatial correlation of complex amplitudes makes the detection performance superior to that of either using raw signal matching [13], [14] or image matching [15].

The remainder of this letter is structured as follows. In Section II, the TWR signal model and the canonical corner response are introduced. Section III presents the proposed correlogram-based strategy together with other matching techniques for corner detection. Supporting results are provided in Section IV, and Section V states the conclusion.

\section{TWR Signal MOdEL}

We consider a monostatic $N$-element synthetic line array. It is noted that the concept can easily be extended to 2-D and/or bistatic arrays. We assume that the data acquisition is carried out in an oblique position, as shown in Fig. 1(a), which significantly attenuates the wall returns and enhances corner scatterers. Let the $n$th transceiver illuminate the scene with a stepped-frequency signal consisting of $M$ frequencies. The response of the scene can be modeled as the sum of responses from individual scatterers, assuming that the scatterers do not 


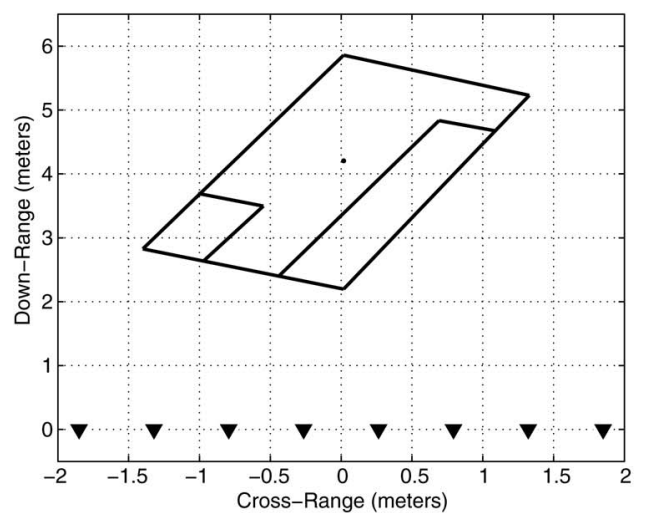

(a)

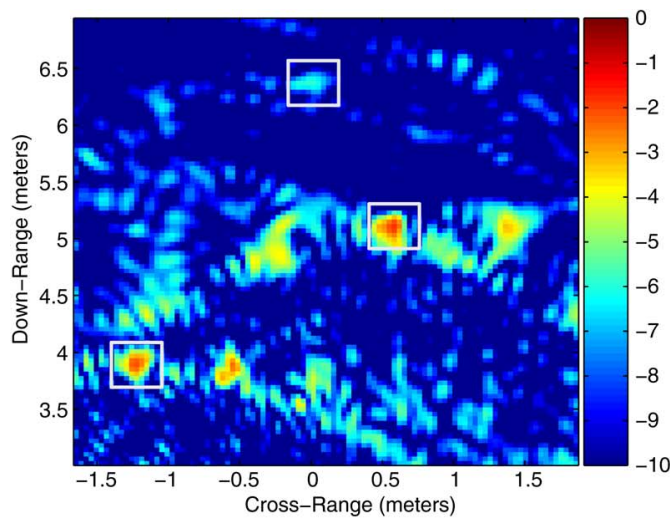

(b)

Fig. 1. (a) Geometry of the simulated scene. (b) Backprojection image of the scene considering the full data volume.

interact with each other. Thus, the signal received by the $n$th transceiver at the $m$ th frequency can be represented as

$$
y(m, n)=\sum_{p=1}^{P} S_{p}\left(m, n, \bar{\phi}_{p}\right) e^{-j \omega_{m} \tau_{p, n}}+w(m, n)
$$

where $P$ is the number of corner scatterers present in the illuminated scene, $\tau_{p, n}$ is the two-way traveling time of the signal from the $n$th antenna to the $p$ th corner scatterer, and the $m$ th frequency $\omega_{m}$ is defined as

$$
\omega_{m}=\omega_{0}+m \Delta_{\omega}, \quad m=0, \ldots, M-1
$$

with $\omega_{0}$ and $\Delta_{\omega}$ denoting the lowest frequency in the bandwidth spanned by the stepped-frequency signal and the frequency step size, respectively. The term $w(m, n)$ in (1) models the contributions of scatterers other than corners, including the walls, humans, and possible multipath propagation effects. The canonical scattering response $S_{p}\left(m, n, \bar{\phi}_{p}\right)$ of the $p$ th corner (dihedral) reflector with orientation angle $\bar{\phi}_{p}$ is given by [16]

$$
S_{p}\left(m, n, \bar{\phi}_{p}\right)=A_{p} \operatorname{sinc}\left(\omega_{m} \frac{L_{p}}{c} \sin \left(\phi_{p, n}-\bar{\phi}_{p}\right)\right)
$$

where the variables $A_{p}, L_{p}$, and $\phi_{p, n}$, respectively, define the amplitude, the length, and the aspect angle associated with the $p$ th corner reflector and the $n$th antenna.

A radar image is generated from the $M N$ observations, i.e., $y(m, n), m=0, \ldots, M-1, n=0, \ldots, N-1$, using frequency-domain backprojection as follows. The scene being imaged is partitioned into a finite number of pixels, i.e., $N_{x} \times$ $N_{z}$, in cross range and downrange. That is, the scene can be represented by the complex reflectivity function $r(k, l), k=$ $0, \ldots, N_{x}-1 ; l=0, \ldots, N_{z}-1$. The complex composite signal, corresponding to the $(k, l)$ th pixel, is obtained by applying a set of focusing delays $\tau_{(k, l), n}$ to align all signal returns from the $(k, l)$ th pixel, and then summing the following results: [17]

$$
r(k, l)=\frac{1}{M N} \sum_{n=0}^{N-1} \sum_{m=0}^{M-1} y(m, n) e^{j \omega_{m} \tau_{(k, l), n}} .
$$

Note that the focusing delay $\tau_{(k, l), n}$ corresponds to the twoway travel time between the $n$th antenna location and the $(k, l)$ th pixel. The process described by $(4)$ is performed for all $N_{x} \times N_{z}$ pixels to generate the image of the scene. In essence, backprojection solves the inverse scattering problem of recovering the unknown scene $r(k, l), k=0, \ldots, N_{x}-1$; $l=0, \ldots, N_{z}-1$, from the radar observations $y(m, n), m=$ $0, \ldots, M-1 ; n=0, \ldots, N-1$.

\section{Corner Detection Strategies}

This section describes different matching techniques for estimation of the locations of the corner scatterer. Corners appear in building structures as a result of the right-angle intersection between two walls. Recently, overcomplete dictionaries for sparse representation of corners from compressed observations have been proposed [13], [14], whose atom coefficients directly indicate the presence of building features at specific positions. Alternatively, image-based complex matched filters were proposed for image feature extraction under full data volume [15]. We first review these two approaches and then present the proposed correlogram matching procedure, where the prior knowledge of the corner scattering model is used as a correlogram template or reference for corner recognition.

\section{A. Overcomplete Dictionary for Sparse Scene Representation}

We assume that the corners can be located at any pixel of the image and all corners have the same orientation angle, which is determined by the oblique illumination under consideration. Typically, only a small number of corner reflectors of a particular orientation are present inside a building. As such, they occupy only a few image pixels, rendering the scene as sparse. A dictionary $\boldsymbol{\Lambda}$ based on possible dihedral locations can be then introduced with $N_{x} N_{z}$ columns. Each column is based on the response of a corner located at the corresponding pixel position. The corner response is modeled following (3). The linear relationship between the underlying corner scattering map, represented by the column vector $\nu$ of length $N_{x} N_{z}$ and by the data measurement vector $\mathbf{y}$ of length $M N$, is given by

$$
\mathbf{y}=\Lambda \nu
$$

Consider only $Q \ll M N$ linear nonadaptive samples of $\mathbf{y}$, i.e., $\breve{\mathbf{y}}=\boldsymbol{\Phi} \mathbf{y}$, where $\boldsymbol{\Phi}$ is the measurement matrix that defines the compressed measurement strategy. Then, CS recovers the 
sparse vector $\nu$ from $\breve{y}$ by solving the following optimization problem:

$$
\min _{\nu}\|\boldsymbol{\nu}\|_{1} \quad \text { subject to } \quad \breve{\mathbf{y}} \approx \boldsymbol{\Phi} \boldsymbol{\Lambda} \boldsymbol{\nu}
$$

where $\|\boldsymbol{\nu}\|_{1}=\sum_{i}\left|\nu_{i}\right|$. Several methods are available in the literature to solve the optimization problem in (6), such as basis pursuit techniques [18]-[20] and greedy iterative methods [21], [22]. Note that each element of $\boldsymbol{\nu}$ directly indicates the presence of a corner reflector at the corresponding location.

\section{B. Image-Based Matched Filter}

In this approach, the reference image, obtained from the canonical corner data model, is directly matched to the backprojection image obtained with the observations [15].

Let the complex amplitude values of the illuminated scene image obtained from the compressed measurements be denoted $\breve{\mathbf{r}}$, and the values of the image corresponding to the compressed measurements of a canonical corner at position $(k, l)$ be represented by $\breve{\mathbf{r}}_{(k, l)}^{\mathrm{ref}}$. Then, the corner detection problem can be expressed as

$$
\min _{\beta(k, l)}\left\|\breve{\mathbf{r}}-\beta(k, l) \breve{\mathbf{r}}_{(k, l)}^{\mathrm{ref}}\right\|_{2}^{2}
$$

where $\beta(\cdot, \cdot)$ is a weighted corner indication function, i.e., the nonzero values of $\beta(k, l)$ indicate the presence of a corner at the $(k, l)$ th pixel. The solution to (7) is given by

$$
\beta(k, l)=\frac{\left(\breve{\mathbf{r}}_{(k, l)}^{\mathrm{ref}}\right)^{H} \breve{\mathbf{r}}}{\left(\breve{\mathbf{r}}_{(k, l)}^{\mathrm{ref}}\right)^{H} \breve{\mathbf{r}}_{(k, l)}^{\mathrm{ref}}} .
$$

\section{Correlogram Matching}

We propose the use of the correlogram for corner scatterer detection. For convenience, we use $L_{\infty}$-norm to measure the distance between pixels, i.e., we define the distance between the pixels $\left(k_{1}, l_{1}\right)$ and $\left(k_{2}, l_{2}\right)$ as $\max \left\{\left|k_{1}-k_{2}\right|,\left|l_{1}-l_{2}\right|\right\}$. For a reference pixel, each distance defines a set of pixels equidistant from that pixel. Let the complex amplitudes corresponding to the set of $N_{p}$ pixels located at distance $d$ from the $(k, l)$ th pixel be denoted $\breve{r}^{(d)}\left(k_{i}, l_{i}\right), i=1, \ldots, N_{p}$. Then, the correlogram of the $(k, l)$ th pixel is defined as

$$
\gamma_{(k, l)}^{(d)} \triangleq \frac{1}{N_{p}} \sum_{i=1}^{N_{p}} \breve{r}(k, l) \operatorname{conj}\left(\breve{r}^{(d)}\left(k_{i}, l_{i}\right)\right)
$$

where $\operatorname{conj}(\cdot)$ is the complex conjugate function. From (9), we can build the correlogram matrix as [23]

$$
\triangleq\left[\begin{array}{cccc}
\operatorname{conj}\left(\gamma_{(k, l)}^{(d=0)}\right) & \gamma_{(k, l)}^{(d=1)} & \cdots & \gamma_{(k, l)}^{(d=D-1)} \\
\operatorname{conj}\left(\gamma_{(k, l)}^{(d=1)}\right) & \operatorname{conj}\left(\gamma_{(k, l)}^{(d=0)}\right) & \cdots & \gamma_{(k, l)}^{(d=D-2)} \\
\vdots & \vdots & \ddots & \vdots \\
\operatorname{conj}\left(\gamma_{(k, l)}^{(d=D-1)}\right) & \operatorname{conj}\left(\gamma_{(k, l)}^{(d=D-2)}\right) & \cdots & \operatorname{conj}\left(\gamma_{(k, l)}^{(d=0)}\right)
\end{array}\right]
$$

where $D$ determines the dimension of the matrix. Note that $\hat{\mathbf{R}}_{(k, l)}$ is positive semi-definite by definition.

The isolated presence of a canonical corner in the $(k, l)$ th pixel will generate a particular reference correlogram matrix, which is denoted $\mathbf{R}_{(k, l)}^{\mathrm{ref}}$. The proposed procedure is based on a scan that reacts only when the reference scatterer is present. Based on this assumption, an error function is required to measure the reference corner contribution contained in the given sample correlogram matrix of each pixel. An estimate of the corner intensity level $\kappa(k, l)$ can be formulated as

$$
\min _{\kappa(k, l) \geq 0} \Psi\left(\hat{\mathbf{R}}_{(k, l)}, \kappa(k, l) \mathbf{R}_{(k, l)}^{\mathrm{ref}}\right)
$$

where $\Psi(\cdot, \cdot)$ is an error function between the two matrices. One choice for $\Psi(\cdot, \cdot)$ is the Frobenius norm of the residual correlogram matrix $\hat{\mathbf{R}}_{(k, l)}-\kappa(k, l) \mathbf{R}_{(k, l)}^{\mathrm{ref}}$. The major criticism of this choice is that it does not preserve the positive semi-definite character of the residual correlogram matrix. The manifold of positive semi-definite matrices is a cone, whose points are connected by exponential paths and only locally resemble a flat Euclidean space [24]. As such, the Frobenius norm provides results similar to that obtained with (8).

The residual correlogram matrix must maintain its positive semi-definite property since it is still a correlogram matrix. A more proper detector that best suits the space generated by correlogram matrices is derived by forcing a positive semi-definite residual correlogram matrix. The problem can be formulated as

$$
\begin{aligned}
\max _{\kappa(k, l) \geq 0} & \kappa(k, l) \\
\text { s.t. } & \hat{\mathbf{R}}_{(k, l)}-\kappa(k, l) \mathbf{R}_{(k, l)}^{\mathrm{ref}} \succeq 0 .
\end{aligned}
$$

If $\hat{\mathbf{R}}_{(k, l)}-\kappa(k, l) \mathbf{R}_{(k, l)}^{\mathrm{ref}}$ must be positive semi-definite, so must be $\mathbf{I}-\kappa(k, l) \hat{\mathbf{R}}_{(k, l)}^{-1} \mathbf{R}_{(k, l)}^{\mathrm{ref}}$. Thus, using the eigendecomposition of $\hat{\mathbf{R}}_{(k, l)}^{-1} \mathbf{R}_{(k, l)}^{\mathrm{ref}}$ defined by $\mathbf{U} \Lambda \mathbf{U}^{H}$, we have

$$
\mathbf{I}-\kappa(k, l) \mathbf{U} \Lambda \mathbf{U}^{H} \succeq 0 \Rightarrow \mathbf{I}-\kappa(k, l) \Lambda \succeq 0
$$

where $\Lambda$ is a diagonal matrix of the eigenvalues of $\hat{\mathbf{R}}_{(k, l)}^{-1} \mathbf{R}_{(k, l)}^{\mathrm{ref}}$, and $\mathbf{U}$ is the unitary matrix containing the eigenvectors of $\hat{\mathbf{R}}_{(k, l)}^{-1} \mathbf{R}_{(k, l)}^{\mathrm{ref}}$. Note that, if (13) is satisfied for the maximum eigenvalue, then it is satisfied for all eigenvalues. Therefore, the condition that always ensures positive semi-definite residual correlogram is given by

$$
\lambda_{\max }^{-1}\left(\hat{\mathbf{R}}_{(k, l)}^{-1} \mathbf{R}_{(k, l)}^{\mathrm{ref}}\right)-\kappa(k, l)=0 .
$$

Thus, the solution to (12) is given by the inverse of the maximum eigenvalue of $\hat{\mathbf{R}}_{(k, l)}^{-1} \mathbf{R}_{(k, l)}^{\mathrm{ref}}$. That is

$$
\kappa_{M}(k, l)=\lambda_{\max }^{-1}\left(\hat{\mathbf{R}}_{(k, l)}^{-1} \mathbf{R}_{(k, l)}^{\mathrm{ref}}\right) .
$$

The values of $\kappa_{M}(k, l), k=0, \ldots, N_{x}-1 ; l=0, \ldots, N_{z}-1$ directly indicate the presence of a potential corner at a given pixel location. 


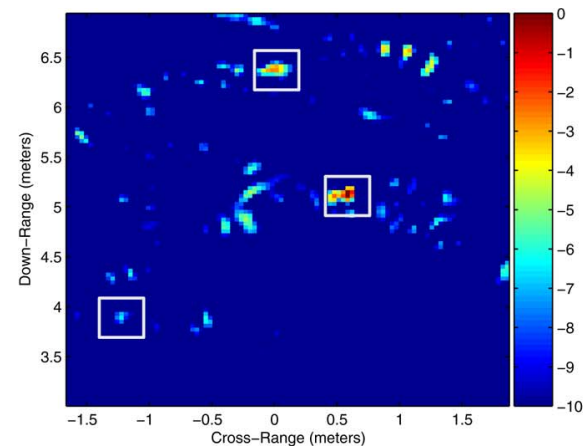

(a)

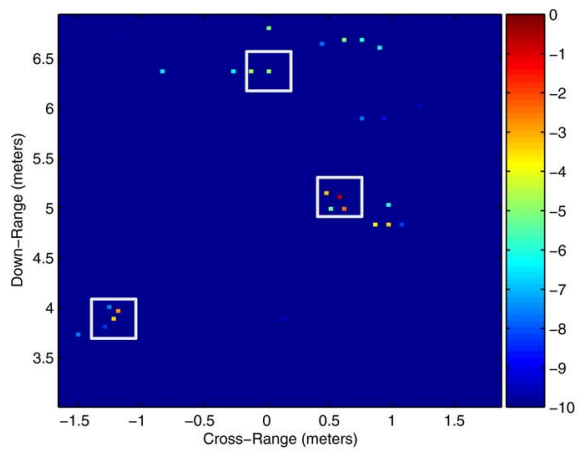

(b)

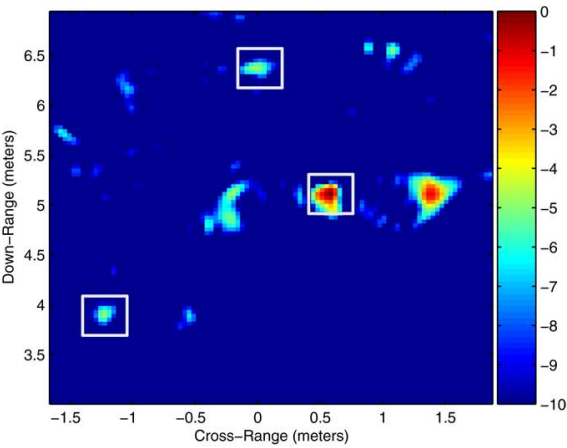

(c)

Fig. 2. Resulting image for $\rho_{f}=0.5$. (a) Correlogram matching $-\kappa_{M}(k, l)$. (b) CS-based reconstruction using overcomplete dictionary. (c) image-based matched filter.

\section{NumERICAL RESUlTS}

In this section, we present results based on numerical EM data. The EM response of a three-room building was generated with FEKO Geometric Optics solver in conjunction with the OPTFEKO options [25]. The simulated scene geometry is depicted in Fig. 1(a). The walls are $20 \mathrm{~cm}$ thick and made of solid concrete with permittivty $=6$. A perfect electric conductor sphere with a diameter of $15 \mathrm{~cm}$ is located at $(0.02,4.24) \mathrm{m}$. A stepped-frequency signal consisting of 201 frequencies covering the frequency band of 1-2 GHz was used for interrogating the scene. An 8-element monostatic line array with an interelement spacing of $53 \mathrm{~cm}(3.53 \lambda$, where $\lambda$ is the wavelength at $2 \mathrm{GHz}$ ) was used. Conventional monostatic operation assumes an antenna spacing smaller than $0.25 \lambda$ in order to avoid grating lobes within the visible region of $-90^{\circ}-+90^{\circ}$. Therefore, the antenna compression rate is $\rho_{n}=0.25 \lambda / 3.53 \lambda=0.07$.

An oblique illumination of the scene is used to avoid wall returns while preserving the important corner features. The angular tilt of the array baseline is $25^{\circ}$. In this case, only the upper left corners of the three rooms are expected to produce strong scattering responses for most, if not all, of the antenna elements. The concave sides of the remaining corners are either facing away from the array or only visible to a small number of antenna elements. We, therefore, focus on the detection of these three corners.

The region to be imaged is chosen to be 3.5 (cross range) $\times$ 3.9 (downrange) $\mathrm{m}^{2}$, centered at $(0.11,4.97) \mathrm{m}$, and is divided into $100 \times 100$ pixels. Fig. 1 (b) shows the backprojection image corresponding to the measured scene, using all 201 frequencies. In this figure and all subsequent images in this section, we plot the image intensity with the maximum intensity value in each image normalized to $0 \mathrm{~dB}$. Although the corners of interest are visible in the image (indicated by white rectangles), it is difficult to discriminate them from other scatterers and clutter even when the full data set is considered. Note that the imaged scatterers appear at biased locations compared with that in the ground truth. This is because the change in propagation speed within the walls has not been accounted for in backprojection.

For corner detection with reduced data, we consider only 101 uniformly selected frequencies. Therefore, the frequency compression rate is $\rho_{f}=101 / 201=0.5$. Fig. 2(a) shows the image obtained with the proposed correlogram matching approach.
TABLE I

TCR FOR $\rho_{f}=0.50$

\begin{tabular}{|c|c|c|c|}
\hline Method & $\mathbf{D = 5}$ & $\mathbf{D}=\mathbf{1 5}$ & $\mathbf{D}=\mathbf{3 0}$ \\
\hline \hline Backprojection & \multicolumn{3}{|c|}{$1.48 \mathrm{~dB}$} \\
\hline CS-based reconst. & \multicolumn{3}{|c|}{$5.41 \mathrm{~dB}$} \\
\hline Image-based match. & \multicolumn{3}{|c|}{$2.66 \mathrm{~dB}$} \\
\hline$\kappa_{M}(k, l)$ & $6.11 \mathrm{~dB}$ & $8.28 \mathrm{~dB}$ & $0.76 \mathrm{~dB}$ \\
\hline
\end{tabular}

More specifically, Fig. 2(a) depicts the values of $\kappa_{M}(k, l)$ for $D=5$. Clearly, the image shown in Fig. 2(a) has detected the three corners and is less cluttered than the full-data image in Fig. 1(b). For comparison, Fig. 2(b) and (c) shows the results obtained with the CS-based reconstruction described in Section III-A and the image-based matching approach of Section III-B, respectively. Orthogonal matching pursuit [26] was used for CS-based reconstruction, with the total number of iterations set to 200. Although Fig. 2(b) provides a relatively clean image with few dominant pixels, some of the strongest ones are outside the white rectangles indicating the corner locations. Fig. 2(c) also has higher intensity clutter pixels compared with Fig. 2(a), which underscores the importance of the positive semi-definite correlogram residual matrix condition.

The superior performance of the proposed technique in terms of scatterer discrimination is confirmed by a comparison of the target-to-clutter ratio (TCR), provided in Table I. The TCR of an image $I(k, l)$ is defined as the ratio between the highest pixel intensity value of the true corner location area $A_{t}$ to the maximum pixel intensity value of the clutter area, $A_{c}$ and can be expressed as

$$
\operatorname{TCR}(I(k, l))=20 \log _{10}\left(\frac{\max _{(k, l) \in A_{t}}|I(k, l)|}{\max _{(k, l) \in A_{c}}|I(k, l)|}\right) .
$$

The corner area $A_{t}$ contains the highest pixel values of the different detected corner areas, which are manually selected in close vicinity to the true upper left corner positions $(11 \times$ 11 pixel rectangular box centered at the real corner position). The clutter area consists of the remaining pixels that are not included in the detected corner areas. Smaller values of the TCR would increase the chance of misclassifications in subsequent thresholding-based detection schemes [27], [28]. In Table I, we observe that the proposed method significantly surpasses the 
TCR values of its counterparts for $D=5$ and 15 , leading to an enhanced detection performance. The performance of the proposed method is clearly linked to the dimensionality $D$ of the correlogram matrix, which should be sufficiently large to capture the image-domain response of a corner reflector and small enough not to infringe over neighboring corners. Increasing $D$ adds more information to the correlogram matrix, resulting in higher TCR values, as shown in Table I for $D=5$ and $D=15$. However, a higher $D$ not only increases the computational complexity but also invades neighboring scatterers, resulting in lower TCR values, as shown in Table I for $D=30$.

\section{CONCLUSION}

In this letter, we have developed a correlogram matching corner detector for TWRI applications under reduced data volume. The correlogram of the scattering response of an isolated canonical corner reflector, which is known a priori, was compared with the correlogram of the received radar image within a correlation matching framework. Results based on numerical EM data demonstrated that the proposed method effectively detects the corner reflectors and outperforms its backprojection imaging $L_{1}$-based reconstruction using overcomplete dictionaries, and image-based matched filter detector counterparts.

\section{REFERENCES}

[1] M. G. Amin and F. Ahmad, "Wideband synthetic aperture beamforming for through-the-wall imaging," IEEE Signal Process. Mag., vol. 25, no. 4, pp. 110-113, Jul. 2008.

[2] M. G. Amin, Ed., Through-the-Wall Radar Imaging. Boca Raton, FL, USA: CRC Press, 2011.

[3] M. Amin and K. Sarabandi, Eds., "Special issue on remote sensing of building interior," IEEE Trans. Geosci. Remote Sens., vol. 47, no. 5, pp. 1270-1420, May 2009.

[4] F. Soldovieri and R. Solimene, "Through-wall imaging via a linear inverse scattering algorithm," IEEE Geosci. Remote Sens. Lett., vol. 4, no. 4, pp. 513-517, Oct. 2007.

[5] H. Burchett, "Advances in through wall radar for search, rescue and security applications," in Proc. Inst. Eng. Tech. Conf. Crime Security, London, U.K., Jun. 2006, pp. 511-525.

[6] F. Soldovieri, R. Solimene, and R. Pierri, "A simple strategy to detect changes in through the wall imaging," Progr. Electromagn. Res. M, vol. 7 , pp. 1-13, 2009.

[7] J. Moulton, S. Kassam, F. Ahmad, and M. Amin, "Target and change detection in synthetic aperture radar sensing of urban structures," in Proc. IEEE Radar Conf., Rome, Italy, May 2008, pp. 1-6.

[8] F. Ahmad and M. Amin, "Through-the-wall human motion indication using sparsity-driven change detection," IEEE Trans. Geosci. Remote Sens., vol. 51, no. 2, pp. 881-890, Feb. 2013.
[9] P. Sevigny and D. DiFilippo, "A multi-look fusion approach to throughwall radar imaging," presented at the IEEE Radar Conf., Ottawa, ON, Canada, Apr./May 2013.

[10] N. Subotic, E. Keydel et al., "Parametric reconstruction of internal building structures via canonical scattering mechanisms," in Proc. IEEE ICASSP, Las Vegas, NV, USA, 2008, pp. 5189-5192.

[11] E. Ertin and R. Moses, "Through-the-Wall SAR attributed scattering center feature estimation," IEEE Trans. Geosci. Remote Sens., vol. 47, no. 5, pp. 1338-1348, May 2009

[12] J. Huang, S. Kumar, M. Mitra, W. Zhu, and R. Zabih, "Image indexing using color correlograms," in Proc. IEEE Conf. Comput. Vis. Pattern Recog., San Juan, Puerto Rico, Jun. 1997, pp. 762-768.

[13] W. van Rossum, J. de Wit, and R. Tan, "Radar imaging of building interiors using sparse reconstruction," in Proc. EuRAD, Amsterdam, Netherlands, Oct./Nov. 2012, pp. 30-33.

[14] E. Lagunas, M. Amin, F. Ahmad, and M. Najar, "Determining building interior structures using compressive sensing," J. Electron. Imag., vol. 22, no. 2, p. 021003, 2013.

[15] M. Greitans, M. Pudzs, and R. Fuksis, "Object analysis in images using complex 2D matched filters," in Proc. IEEE Region 8 Conf. EUROCON, Saint-Petersburg, Russia, May 2009, pp. 1392-1397.

[16] M. Gerry, L. Potter, I. Gupta, and A. van der Merwe, "A parametric model for synthetic aperture radar measurements," IEEE Trans. Antennas Propag., vol. 47, no. 7, pp. 1179-1188, Jul. 1999.

[17] F. Ahmad, G. J. Frazer, S. A. Kassam, and M. G. Amin, "Design and implementation of near-field, wideband synthetic aperture beamformers," IEEE Trans. Aerosp. Electron. Syst., vol. 40, no. 1, pp. 206-220, Jan. 2004.

[18] E. J. Candes, J. Romberg, and T. Tao, "Robust uncertainty principles: Exact signal reconstruction from highly incomplete frequency information," IEEE Trans. Inf. Theory, vol. 52, no. 2, pp. 489-509, Feb. 2006.

[19] E. J. Candes and T. Tao, "Near optimal signal recovery from random projections: Universal encoding strategies," IEEE Trans. Inf. Theory, vol. 52, no. 12 , pp. 5406-5425, Dec. 2006

[20] S. S. Chen, D. L. Donoho, and M. A. Saunders, "Atomic decomposition by basis pursuit," SIAM J. Sci. Comput., vol. 20, no. 1, pp. 33-61, 1999.

[21] S. Mallat and Z. Zhang, "Matching pursuit with time-frequency dictionaries," IEEE Trans. Signal Process., vol. 41, no. 12, pp. 3397-3415, Dec. 1993.

[22] J. A. Tropp, "Greed is good: Algorithmic results for sparse approximation," IEEE Trans. Inf. Theory, vol. 50, no. 10, pp. 2231-2242, Oct. 2004.

[23] M. Shaghaghi and S. Vorobyov, "Correlogram for undersampled data Bias and variance analysis," in Proc. IEEE Int. Conf. Acoust., Speech Signal Process., Kyoto, Japan, Mar. 2012, pp. 3513-3516.

[24] R. Hill, "On the cone of positive semidefinite matrices," Linear Algebra Appl., vol. 90, pp. 81-88, 1987.

[25] FEKO EM Simulation Software Web page. [Online]. Available: http:// www.feko.info

[26] J. A. Tropp and A. C. Gilbert, "Signal recovery from random measurements via orthogonal matching pursuit," IEEE Trans. Inf. Theory, vol. 53, no. 12 , pp. 4655-4666, Dec. 2007.

[27] C. Debes, M. G. Amin, and A. M. Zoubir, "Target detection in singleand multiple-view through-the-wall radar imaging," IEEE Trans. Geosci. Remote Sens., vol. 47, no. 5, pp. 1349-1361, May 2009.

[28] C. Seng, M. Amin, F. Ahmad, and A. Bouzerdoum, "Image segmentations for through-the-wall radar target detection," IEEE Trans. Aerosp. Electron. Syst., vol. 49, no. 3, pp. 1869-1896, Jul. 2013. 